\title{
SBS Korea: A fully asset-management based digital news operation in action
}

\author{
Charles Bebert \\ has a background in engineering and software design. Kane specializes in benchmark studies and best practices, \\ publishing "rolling studies" on HD, server-based news, media asset management and market sizing. Charles is currently \\ working as consultant in 16 countries including the USA, Korea, UK, France and Belgium.
}

Keywords: video, SBS, Konan, Korea, Kane, broadcast, case study

\begin{abstract}
Kane, a market research company based in Paris, has developed a series of reports on server-based news operations and media asset management (MAM) systems in the broadcast sector. These studies, updated twice yearly, are based on several hundreds of site visits with leading broadcasters and manufacturers. They analyze and track developments in functionality, best-practice and return on investment, and provide a strong basis for carrying out detailed evaluations of news and media asset management solutions. The studies and the body of data built up over the years by Kane are the starting point for this comparative case study of the technology solutions in operation at SBS, one of the leading broadcasters in the AsiaPacific region. The report shows that, according to the best practice criteria established by Kane, SBS is at the very top of the global scale and operating at a level of effectiveness which is higher than any other broadcaster studied by Kane.
\end{abstract}

\section{INTRODUCTION}

For some years, digital asset management has been a hot topic for broadcasters. Almost all television and radio enterprises, wherever they are in the world, face the same twin imperatives: cut costs by improving efficiency, and generate new revenue by exploiting new content delivery opportunities, such as video on demand, internet streaming and content-tomobile. Many concede that the implementation of broadcast-specific

tvbeurope@scope.ie asset management systems is a

prerequisite for meeting both

requirements, but are still unsure of the

right choice of system or

implementation strategy. There have

been a number of less-than successful

trials, and few real successes.
This is why considerable attention is now being paid to the asset-management based news production system in operation at SBS in Korea. SBS is Korea's largest commercial broadcaster, delivering its programs via television (terrestrial, cable, and satellite, and including an HD service) and radio. It is one of three premium channels in Korea, and employs over 1,500 staff.

In December 2003, SBS moved into a new headquarters building, and decided that as part of the process its news operation should migrate to a new technology base, one that would provide SBS with both operational efficiencies and a platform for introducing new services in the future. Central to this concept was a production infrastructure based on disk-based shared storage 
accessible to all, which would allow easy access both to current production material and the archives, while at the same time putting the latest generation of creative tools in the hands of journalists and production staff.

A task force of six people began work in July 2002, and in March 2003 the choice was made to appoint IBM as integrator, and the Korean company Konan, a media asset management (MAM) solution vendor, as the main software provider.

The first news show was broadcast with the system in February 2004 and all bulletins were on the air with the new system by June 2004. The total project duration was exactly two years.

\section{THE SBS NEWS OPERATION}

The SBS newsroom produces news for its terrestrial channel and its web service (SBS also produces three cable channels themed on golf, other sports and drama, with no newscasts).

The main 8 o'clock news lasts for 45 minutes and usually includes upwards of 32 stories, most of which contain a video element. In addition to the main bulletins SBS generates another seven daily bulletins, a total of over four hours of coverage. The newsroom has almost 300 journalists, of whom 120 are reporters in the field, along with 46 cameramen and 48 craft editors as well as ingest, graphic and production staff. These personnel are served by one of the world's largest digital production systems, which includes:

- forty concurrent ingest channels (Konan);

- MAM server (Konan) storing and managing all media assets including workflow and media transfers;
- central digital storage with IBM GPFS providing 300 hours online and 300 hours nearline (total $28 \mathrm{~Tb}$, mirroring system);

- forty-one craft-editing systems (GVG Vibrint) operating at MPEG-2 (50Mbps);

- a dual low-resolution environment in which the Konan system digitizes all incoming material at MPEG-1 (1.5 Mbps) and at 356Kbps using Windows Media 9. MPEG-1 provides for 300 journalists editing at low-resolution while 1,000 users can browse media with Windows Media 9;

- a digital archive of 25,000 hours (FrontPorch Digital DIVArchive and a Storagetek data library);

- Newsroom computer systems (NRCS) provided by CIS, and automation software provided by D2Net, both Korean companies.

Underpinning all of this is a MAM system provided by Konan which provides comprehensive cataloging, retrieval and workflow management capabilities. The Konan environment is built of four main components, all of them developed by Konan:

- Asset manager: This is the principal MAM server, which stores and manages all video content and associated metadata in the system and controls their relationships. It provides workflow management, management of user transactions, utilization and distribution of contents, and traffic control.

- Search engine: This is a flagship product for Konan that supports large-volume multimedia search and a large number of concurrent users in multiple languages. It has been the subject of many years of development (Konan search tools are 
licensed by many IT developers and ISP's in Asia).

- Cataloguer: This is a tool for analyzing video clips and extracting key metadata, such as keyframes, closed captioning and face characters. Keyframes are extracted based on automatic detection of scene changes and image indexing. The metadata are stored in the asset manager server. The Konan Cataloguer supports up to eight times faster than real-time playback for a digitized stream.

- Client modules. Konan provides four different client modules:

- A browser for searching and viewing video with metadata, storyboard, extracted face images and thumbnail images.

- A MetaEditor for viewing and editing metadata.

- A ProxyEditor for rough-cut editing, and

- An administrator module for the management of the entire MAM system and users.

\section{THE SBS ARCHIVE}

Since its launch in 1991, SBS has generated over 70,000 hours of news archive material, which is stored on a total of almost 150,000 cassettes. As part of the run-up to the digital news project, archivists selected and ingested the most important and relevant 3,000 hours of this deep-archive material - they are one of very few broadcasters in the world who have carried out such a systematic digitization of existing tapebased archive material.

Fifteen months into the digital era, the data library now manages almost 9,000 hours of news archive, corresponding to some 70,000 items. SBS has three archivists who catalog "on the fly" at the ingest desk, and five others who select from source materials and rushes

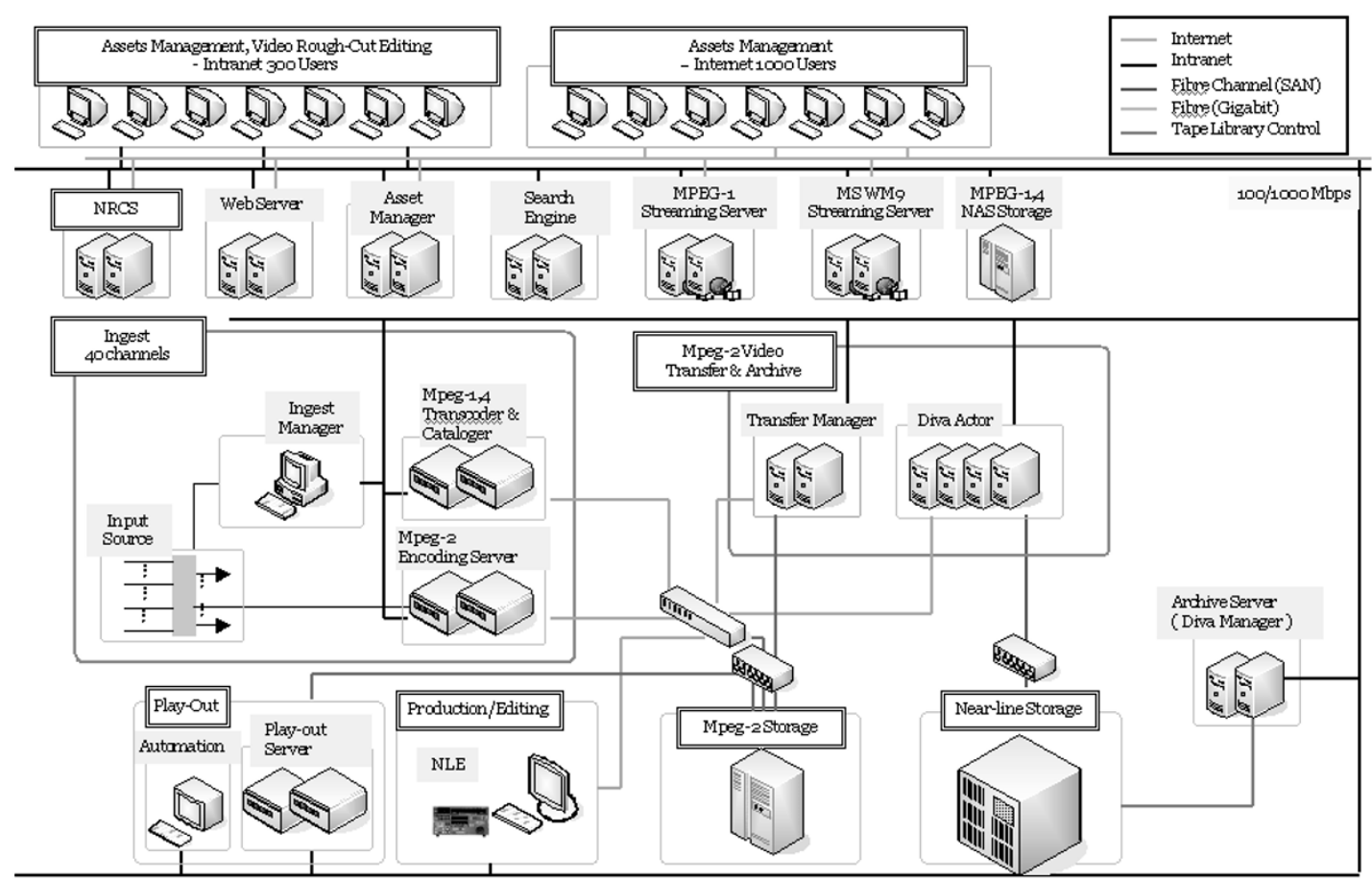

Figure 1: SBS system diagram 
video to be saved in the data library or online. SBS saves an average of 12.5 hours of material per day, including each newscast as a single item, and up to 8.5 hours of source material. SBS no longer keeps any new material on tape other than as data.

\section{BEST PRACTICE ANALYSIS}

Kane has carried out extensive research among broadcasters and manufacturers worldwide and has compiled a digest of best practices for digital news production. These fall into five categories:

- ingest and cataloging;

- production;

- archiving;

- playout and distribution;

- integration and security.

Analysis for SBS shows, in each category, a number of issues, their impact and how they are addressed in the SBS digital system.

\section{Ingest and cataloging}

Best practice ingest requires a number of features in order to capture live feeds from a variety of agencies with as high degree of automation as possible. These include scheduled as well as "crash" records, segmentation of agency feeds into individual stories. The ability to use LAN and WAN technologies are all notable features. There are considerable potential savings to be realized by replacing a legacy video distribution system with an IP-based WAN.

Streaming or chunking increases the speed of processing by allowing breaking news to begin to be edited even before transmission of rushes is over.
Automatic indexing addresses the high cost of human cataloging and allows a more efficient archiving process, although a degree of manual indexing is unavoidable. A thesaurus can take time for an archivist to build, maintain and use, and many broadcasters do without one; however, a "keep it simple" thesaurus is extremely effective in certain cases to enhance search efficiency and speed. Another feature is still barely present among broadcasters although journalists have long asked for it: indexing a video by the text of the voiceover.

In terms of indexing and cataloging, the SBS case is notable because volumes are huge in terms of incoming feeds, and because of the use of a high resolution of $50 \mathrm{Mbps}$. Face detection is a feature unique to Konan, and helps archivists to systematically index by a person's name and face.

\section{Production}

New technical architectures based on networked storage bring to all journalists (at last) the ability to share all media. An appropriate low-resolution capability makes this perfectly possible from the field or from remote locations, and in addition allows other departments to browse media from a standard workstation. Bandwidth is an issue for large enterprises, and a cost for small ones. Dual or triple media databases (ie high resolution, low resolution 1 for editing, low resolution 2 for browsing) add complexity, and can be hard to keep synchronized especially for late-breaking material.

Special effects are generally simple in news, but some are necessary for legal reasons, such as mosaics for concealing identities. Multiskilling (especially 


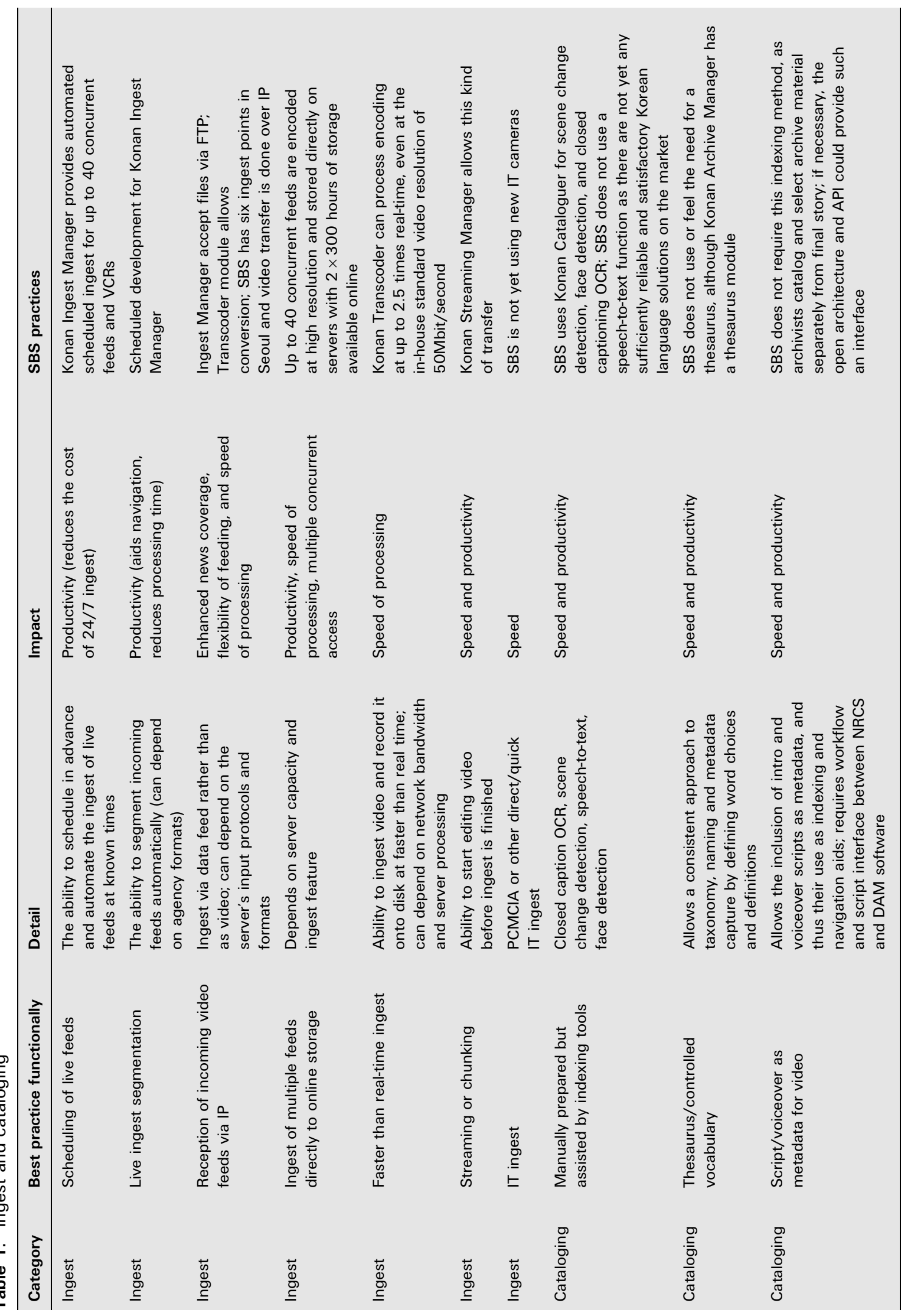


journalist editing) may be considered as best practice, as it provides additional coverage capabilities, and an increase in production capability in the case of breaking news. According to our report, 60 per cent of newsrooms are practicing journalist editing. A smaller proportion

(20 per cent) create and record voiceovers as part of browse-resolution editing.

Graphics are crucial to make news understandable. Creating a graphic is largely an editorial process rather than a production one, which is why templatebased graphics (where the journalist can choose a graphic style from a catalog) and simulation tools are so important.

SBS is notable because of the use of Windows Media 9 as second browse resolution for visualization, and because of a high level, for a premium channel, of multiskilling among journalists. This is still more common for small channels or continuous news channels. At SBS, all news items for the main $8 \mathrm{pm}$ bulletin are rough cut by journalists, to be reviewed and finished by craft editors.

According to a satisfaction survey commissioned by SBS, Mr Lee, Vice President and project manager, noticed that 20 per cent of journalists got used to editing after one month, and 41 per cent after 23 months. It took one year for 6 per cent.

Nine per cent do not feel the need for re-training, 46 per cent would like refresher courses two or three times a year, and 32 per cent once a year.

\section{Archive}

The advent of mass storage on data libraries or on disk arrays accentuates the need for a highly efficient search engine capable of dealing with hundreds of thousands of items with a high level of performance. Many of the best engines were developed for internet search portals, and mark a true convergence between the internet and broadcasting. It is no coincidence that Konan's search engine, which powers the SBS digital news system, is licensed by many Asian internet portals.

Best practice content management in broadcast requires the concept of a "container" which allows broadcasters to group assets in many formats within the same "folder;" not just video and audio tracks, but attached scripts and wires, and other related audio, graphics, animation, and photos.

The rapid emergence of HDTV around the world (in which Korea is a leader) creates a new challenge for preservation at a higher resolution than the $25 \mathrm{Mbps}$ normally considered as sufficient for news. Very few use $50 \mathrm{Mbps}$ as well as IMX, but solutions are now under development by all manufacturers and it is just a question of time.

Very few newsrooms are managing metadata relative to rights clearance as well as inheritance rules when using several sources to edit a final story. However, this is increasingly important.

Most broadcasters maintain a deep archive on shelves, even if they have online storage for current and future material. Once they launch IT-based production systems, they start keeping all or some edited packages and, in certain cases, rushes.

The SBS archive is notable because while it is one of around 50 newsrooms, globally, with an IT-based mass-storage archive, it is one of only a handful to have systematically digitized past news content. Many broadcasters do little 


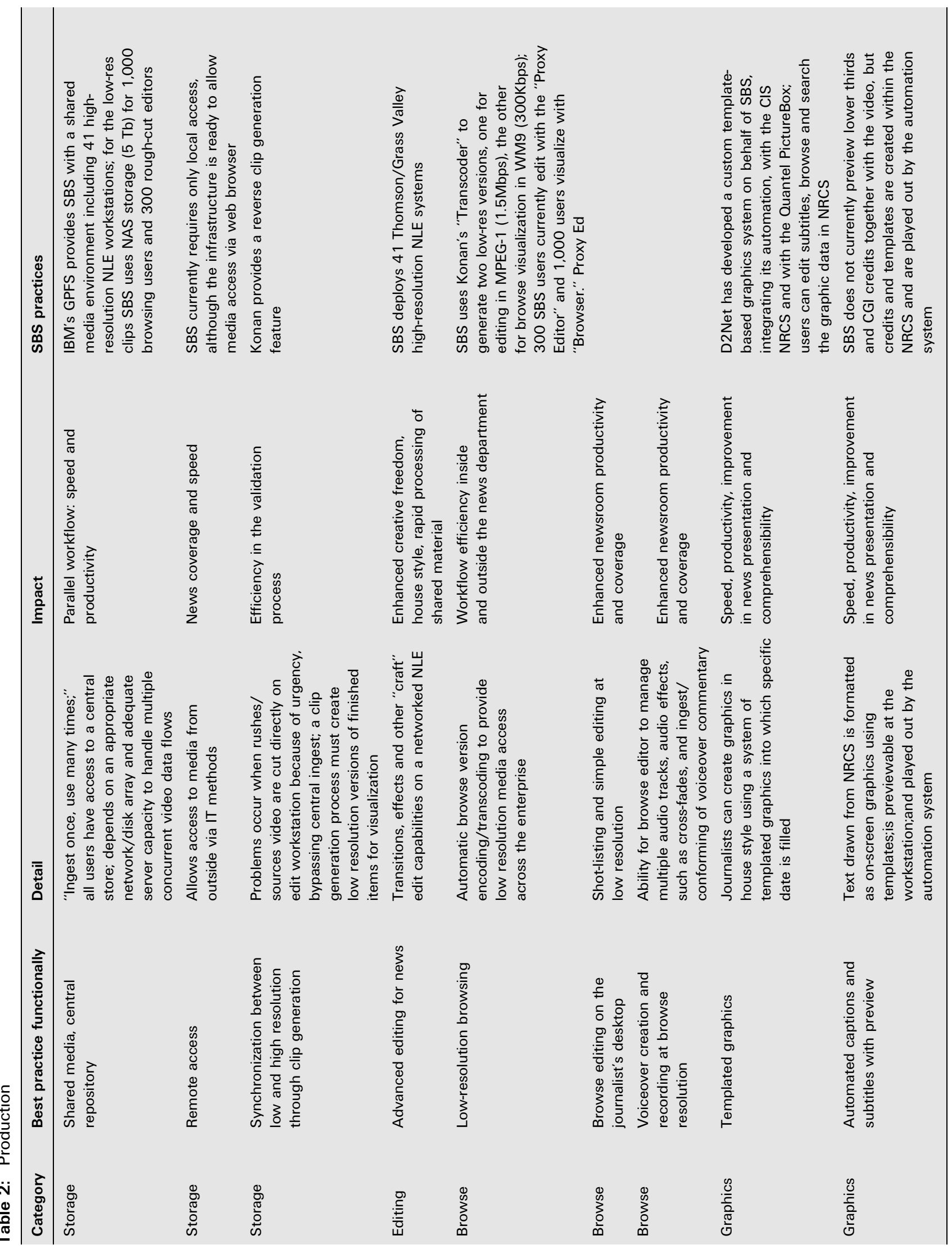




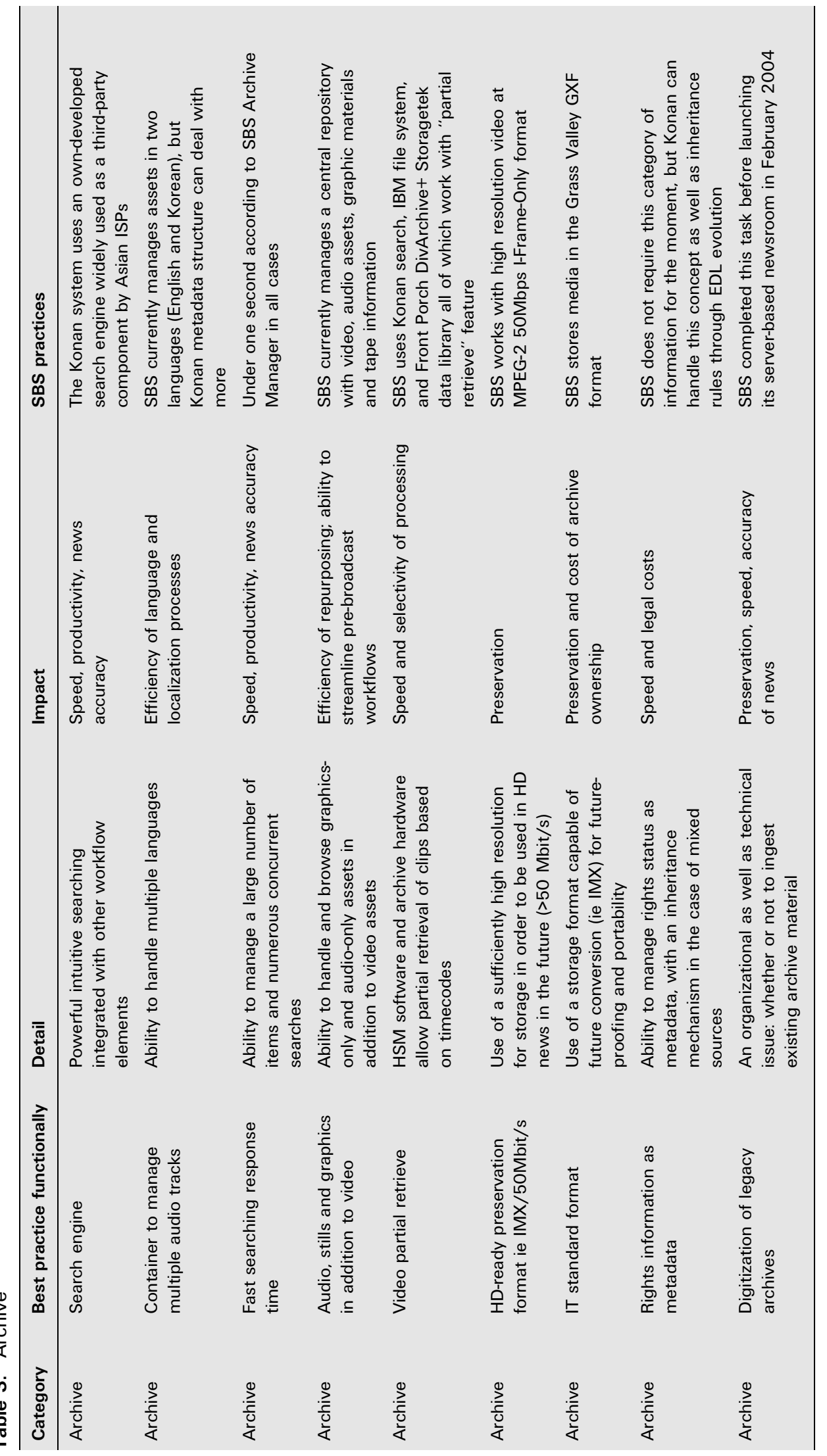


with their historical archives because they do not know the content sufficiently to make a relevant choice. At SBS, the quality of the previous "text" archive database was good enough, and SBS is helped by its relatively short history. SBS is also unusual in that it saves source material selections rather than final stories. Final stories are saved inside bulletins preserved as a whole, rather than individually.

The SBS archive solution has a large online capacity of 600 hours, and relies technically on a GPFS IBM file system which looks robust enough after finetuning for 50Mbps, and on Konan's celebrated search engine, which is a standard in the Unicode world.

\section{Playout and distribution}

Getting breaking news to air as soon as it arrives is a crucial element of best practice.

It is key for productivity and reduction of staff in the news gallery to easily group together (and produce as one element rather than many) video, audio, stills, CGI, graphics and animation, and HTML.

It is highly desirable for certain news formats to be able to create a "news wheel" (ie an automated news bulletin without presenters), particularly for a 24-hour news channel, but also where premium channels are proliferating the number of bulletins without additional staff.

Newsroom computer systems must control automation, either internally or by interfacing seamlessly with thirdparty automation systems. This allows changes at the last moment (to drop or to replace a story) to be decided from a journalistic perspective. The agility of the rundown is increasingly important as bulletins are more and more subject to last-minute changes. Therefore the userfriendliness of the status display inside the rundown is essential.

It is becoming compulsory to have publishing tools (and technology) to repurpose scripts and videos for mobile phones and internet portals.

At SBS, the level of integration of all systems components (Konan browse, ingest, media management and transfer, CIS NRCS, D2Net automation and graphics interface) are very much state of the art in terms of smart interfaces. This allows last-minute changes to be made, and very low staffing levels in the news control room gallery for both short and long newscasts. SBS's use of open standards and XML architecture will allow repurposing of content for other media or other channels when needed in the future.

\section{Integration and security}

As IT-based systems become more and more the norm in broadcasting, integration and security are a more and more pressing issue.

Newsrooms need to assemble many proprietary pieces of hardware and software quickly and efficiently into real-time systems without the need to access or change source code. Operating systems, XML, truly supported APIs, a consistent services infrastructure and a component-based architecture are important best practice steps towards speed of implementation and futureproofing.

Scalability is also important and is closely linked to an open architecture.

Redundancy is seldom in the network area, but progress is being made to secure solutions, with back-up and disaster recovery sites becoming more 


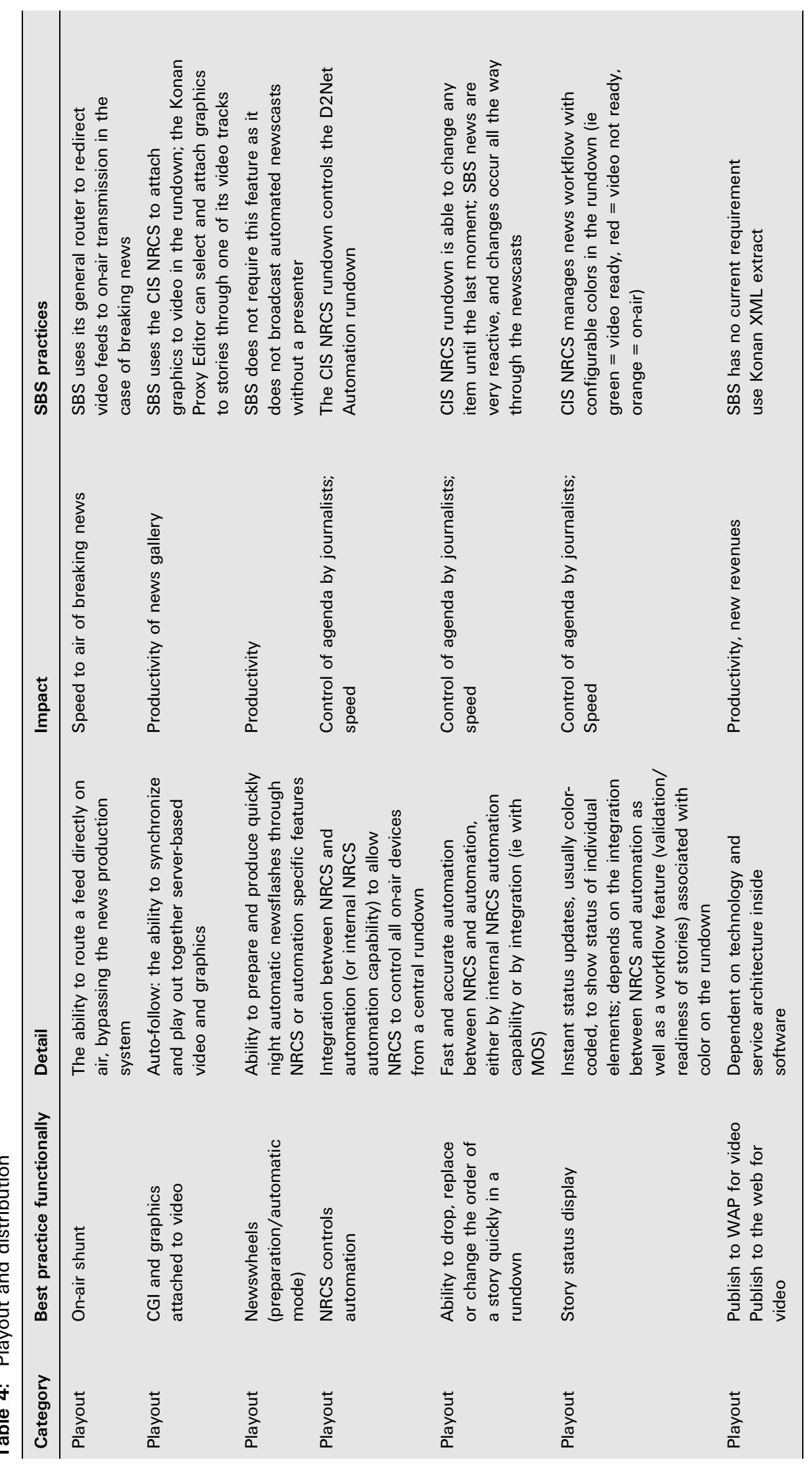


common. In many places, there is a new vision regarding maintenance where IT, broadcast and networking know-how must all be shared by every maintenance staff member.

The SBS implementation is particularly interesting due to the simultaneous launch of new editorial, production and archive solutions after a project gestation of only two years. Most other broadcasters adopt a stage approach which takes much longer. SBS's speed of implementation is also remarkable because a new building and complete technical installation was also created at the same time. The SBS project was the result of a very experienced seven-person team many of whom had backgrounds of 15 years or more in broadcast, IT or journalism. Another crucial factor was that Konan, responsible for much of the software involved, had previously developed SBS's radio archive solution, and Konan itself had a long history of major projects.

\section{BEST PRACTICE CONCLUSIONS}

There is no doubt that the evidence of Kane's best practice analysis places SBS and its suppliers, especially Konan, in a leading position among the world's broadcasters.

We have compared SBS with a number of leading broadcasters in Europe and North America, and while the average broadcaster in this category achieves a score of 59 per cent against our list of best practice elements, SBS achieves 77 per cent.

Many of the areas where SBS is not following our best practice guidelines are a matter of operational and managerial choice, and could be achieved if desired. In fact, SBS could already achieve 91 per cent, and even 95 per cent with the promised 2006 developments from Konan.

According to Kane's evaluation method, therefore, SBS's implementation is one of the best and most advanced in the world.

\section{RETURN ON INVESTMENT}

Return on investment (ROI) is a key element of every major broadcast project, and it is interesting to examine SBS's implementation in that light.

Like many other broadcasters, SBS has not reduced staff by implementing a server-based newsroom. There have been several small adjustments, such as editors (minus 2) and archivists (plus 2). But as a total, staff numbers have remained constant.

On the other hand, SBS currently produces eight newscasts a day, as opposed to seven previously. Actual news production has increased markedly, from 158 minutes per day prior to February 2004, up to 246 minutes by May 2005, an increase of 56 per cent. This result has been obtained in spite of a reduction in the working week for each staff member from six days to five, an additional productivity increase of 20 per cent.

In total, therefore, with static staff numbers, productivity has improved by 87 per cent (combining increases of 56 per cent and 20 per cent) since SBS launched its new server-based and complete archive integrated system.

Without the server-based and desktop technology to edit, broadcast and repurpose news material, SBS would have had to recruit anything up to 179 additional personnel to achieve the same volume increase, against a start level of 


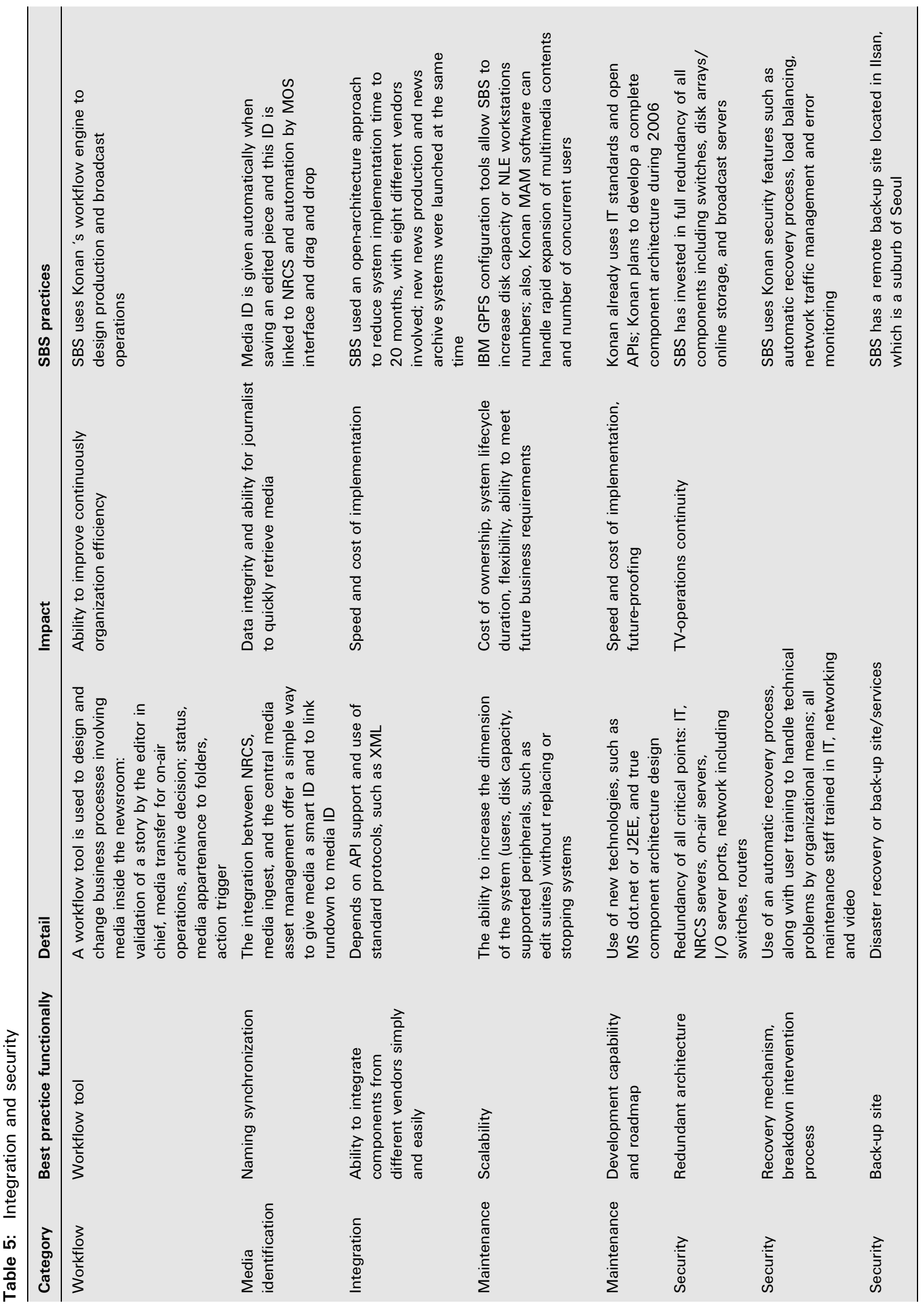


206 staff. Even if we concede that SBS could have made some productivity gains with traditional tape-based newsrooms, it is reasonable (but very conservative) to estimate that only 20 per cent of 179 additional staff would have been necessary to recruit. Average cost saving can thus be estimated to 20 per cent $\times 179 \times \$ 130 \mathrm{k}$ per year.

Another direct gain is realized by SBS avoiding external storage space for the archives. Before launching a digital mass storage system, SBS used to rent an additional 66 square meters of physical storage on shelves, at an accumulating yearly cost of $\$ 40 \mathrm{k}$ per year.

Taking everything into consideration, it is estimated that the SBS's overall investment in the digital news and archive systems will be repaid in three years. With a less conservative hypothesis this could be reduced to little over a year.

This is impressive enough. But the true "jackpot" will come if and when SBS gets authorization from the Korean Broadcast Regulation Authority to launch new channels requiring a news element.

If this can be carried out without having to hire significant additional staff, the incremental advertising revenue can be attributed as direct gains generated by SBS's digital production system.

Harder to assess and validate are the more "qualitative" aspects of the ROI equation at SBS. Examples of these qualitative gains might be streamlined workflow, work efficiencies gained, opportunity cost by not losing or misplacing contents, improved quality of media content thanks to the digitized process and contents management, better coordination among employees along the workflow, and so on.
Although these are hard to measure, the evidence given by SBS staff working on the new system would support a view that such gains are being achieved.

\section{SBS'S RECOMMENDATIONS}

Hanggyu Han, SBS Head of Archives and Sung-Myung Lee, SBS Vice President and overall head of the project, offer a number of

recommendations for other broadcasters contemplating an archive project.

Mr Han says that training and communication are by far the most important success factors.

Mr Lee has compiled the following set of "rules" for a successful project:

1. Communication: be sure to explain fully the aims of the project to users.

2. Partnership: choose your integration company with care: they are your insurance policy.

3. Training: people are the biggest barrier to technology implementation unless they are educated sufficiently.

4. Management commitment: direct access to and support from the CEO is crucial. The management of fundamental change, especially when workflow is involved, means that there will inevitably be moments when CEO support must be used to reinforce the objectives

5. Patience: enough time must be given to a project to succeed. Goals and timetables must be truly realistic and achievable and not cater to unrealistic expectations of users or management.

6. Stabilization: it must be recognized that bugs are inevitable in any software project, and not so much a 
threat to reliability but the means of achieving it as long as they are correctly resolved.

7. Passion: the project team and its patrons must show total commitment to its success.

\section{CONCLUSIONS}

There can be no doubting the success of the newsroom project at SBS. Working in close partnership with its suppliers and notably with the Korean company Konan, SBS has succeeded in implementing highly progressive new operational processes and workflows with a minimum of disruption.

Konan's combination of an extensive existing product base and a willingness to enter wholeheartedly into a development program to meet SBS's needs is a crucial part of the project's impact, and the result is a system which goes further down the road of a fully asset-management based news production and delivery operation than any other broadcaster we have seen.

It is notable that in our formal best practice analysis SBS and Konan score more highly than any other broadcaster we have studied.

Equally impressive is the way that SBS have managed the project, especially the human elements which so often prove difficult. It is obvious that staff at SBS have been fully involved in the project, and well-trained on the new systems.

In both what they have achieved, and how they have achieved it, SBS and Konan deserve widespread recognition. 